\title{
¿Qué es esa cosa llamada intraemprendedor? Gestión del trabajo en el capitalismo cognitivo y concepciones emprendedoras
}

\author{
Antonio Santos-Ortega ${ }^{1}$; David Muñoz-Rodríguez ${ }^{2}$
}

Recibido: 03 de diciembre de 2017 / Aceptado: 06 de abril de 2018

Resumen. El artículo estudia la figura del intraemprendedor en el marco actual de ascenso de los discursos emprendedores en el trabajo. En estos discursos, el intraemprendedor surge como nuevo arquetipo del trabajador asalariado, caracterizado por la implicación y la capacidad de innovación, y se presenta como tipo ideal en el cual ha de proyectarse cualquier empleado. El intraemprendedor es un dispositivo de activación para los asalariados. Los objetivos del artículo consisten en explorar cómo se construye el intraemprendedor desde la literatura empresarial. Para cubrir este objetivo se analizan noticias de prensa, artículos en revistas mensuales de gestión y en revistas académicas del área de economía y empresa. Un segundo objetivo busca enclavar la figura del intraemprendedor en el contexto de cambios en la organización del trabajo durante el posfordismo. Se realizará para ello un análisis crítico de algunos de los principales autores que han abordado la cuestión desde la sociología económica.

Palabras clave: Intraemprendedor; emprendedor; activación; capitalismo cognitivo; posfordismo; trabajo

\section{[en] What Is This Thing Called Intrapreneur? Work management, cognitive capitalism and entrepreneur conception}

\begin{abstract}
This article studies the figure of the intrapreneur in the current framework of the rise of entrepreneurial discourses at work. This figure identifies the salaried worker with entrepreneurial behaviours within his or her company and can even create an ex novo one if the intra-enterprise programs of their company allow it. In the current discourses on business innovation, the intraentrepreneur emerges as a new archetype of the salaried worker, characterized by his or her involvement and the ability to innovate, and presents himself or herself as the ideal type in which any employee should project himself or herself. The intra-entrepreneur is an activation device for employees. The objectives of this article are to explore how the intrapreneur is built on the basis of business literature. To cover this objective, press releases, articles in monthly management magazines and academic journals in the area of economics and business will be analysed. A second objective seeks to place the figure of the intraentrepreneur in the context of changes in the organization of work during the post-Fordism. To this end, a critical analysis will be made of some of the main authors who have approached the issue from the point of view of economic sociology.
\end{abstract}

Keywords: Intrapreneur; entrepreneur; activation; cognitive capitalism; postfordism; work

Sumario: 1. Introducción. 2. El surgimiento del intraemprendedor en la retórica empresarial. 2.1. Aspectos terminológicos previos de la noción de intraemprendedor. 2.2. El intraemprendedor como

\footnotetext{
Dpto. Sociología y Antropología Social. Univitat de. València. juan.a.santos@uv.es

2 Dpto. Sociología y Antropología Social. Univitat de. València. francisco.d.munoz@uv.es
} 
arquetipo del trabajador emprendedor e innovador en la literatura de gestión empresarial. 2.3. El intraemprendedor y sus metáforas: perfiles y rasgos de carácter. 3. La figura del intraemprendedor en el núcleo de los cambios del posfordismo. Una revisión desde las ciencias sociales. 4. Conclusiones. 5. Referencias bibliográficas.

Cómo citar: Santos-Ortega, A.; Muñoz-Rodríguez, D. (2018) “¿Qué es esa cosa llamada intraemprendedor? Gestión del trabajo en el capitalismo cognitivo y concepciones emprendedoras". Cuadernos de Relaciones Laborales, 36(2), 285-303.

\section{Introducción}

La figura del emprendedor se ha convertido en esta última década en el equivalente general de las conductas socioeconómicas, una escala de medida, un canon frente al que compararse. Desde el momento en que alguien se confronta con algunas de las figuras más visibilizadas, excelentes y heroicas de este canon, comienza a desplegarse un código no escrito de comportamientos y protocolos dignos de imitar; un estilo que nos orienta hacia las expectativas sociales más sobresalientes que han de guiarnos para definir nuestros objetivos hoy. En caso de que aún no los tuviésemos, el modelo de conducta que se propone desde la figura del emprendedor los desencadena, los activa, incita a fijarlos porque ya están a la orden del día. A la manera de los eficaces plug and play, el sistema operativo del emprendedor se instala automáticamente e imperceptiblemente en nuestros códigos de apreciación social. La reiteración, la réplica permanente, la difusión ubicua, su continua repetición en boca de todos, de jóvenes deportistas, artistas, científicos, compañeros de aula, presentadores televisivos y, por supuesto, emprendedores, normalizan el mensaje, pudiéndonos llevar a pensar que estamos personalmente muy atrasados en nuestro carácter emprendedor. Los medios de comunicación están copados por personajes que enarbolan la energía emprendedora en su estado puro, ensanchando el mito del emprendedor y todos sus valores: el éxito económico, el conocimiento técnico, el progreso, un espíritu de esfuerzo, el respeto por el medio ambiente o por la justicia social. Blogueros, periodistas, personas influyentes, profesores universitarios construyen un relato sirviéndose de todo este storytelling de valores, arrebatadoramente cool, que se presenta de manera desacomplejada y natural, acelerando nuestra credulidad. Resulta difícil oponerse a esta magnífica escenificación optimista de la vida, sobre todo, cuando la realidad social y económica es cruda y escasa en alternativas.

Aunque, como se deriva del párrafo anterior, el lado más visible del emprendedor hoy es su faceta popular y su espíritu positivo, en realidad, su significación más profunda se desarrolla actualmente en el mundo de la economía y del trabajo. En estos ámbitos, ocupa un lugar central en esta última década ya que se enclava en el contexto de los grandes cambios sociolaborales actuales. En primer lugar, se enclava en el marco del trabajo asalariado tradicional, con una progresiva empresarialización del trabajo asalariado en sí mismo y una incitación a la individualización y la responsabilización, que se visibiliza, sobre todo, en los empleos más cualificados. En segundo lugar, se enclava en la rápida difuminación de las fronteras del trabajo asalariado con la variada proliferación de nuevas figuras laborales basadas en el trabajo por cuenta propia. El capitalismo de plataforma y el digital labor ge- 
neran nuevas prácticas y arquetipos que traerán un profundo impacto en el mundo del trabajo.

En este texto, vamos a concentrarnos en el análisis del primer tipo de cambios. Es decir en las transformaciones que se están produciendo en el seno del trabajo asalariado a raíz de su progresiva empresarialización, de su reconceptualización desde la lógica emprendedora en estos últimos años. En este proceso, el intraemprendedor emerge como nuevo arquetipo del trabajador asalariado. La retórica empresarial rediseña el rol tradicional de asalariado a través de esta categoría de intraemprendedor -un asalariado que emprende dentro de su empresa, un trabajador que se comporta como empresario- y lo presenta como tipo ideal en el cual ha de proyectarse cualquier empleado. Con ello, se pretende redefinir las viejas identidades asalariadas, los viejos enfrentamientos entre el capital y el trabajo, las relaciones laborales, y se trata de introducir nuevos hábitos respecto a la implicación del trabajador, su compromiso en la empresa. El intraemprendedor es un dispositivo de activación para los asalariados. Este artículo tiene el objetivo de explorar cómo se construye el intraemprendedor desde la literatura managerial y cómo actúa sobre la subjetividad de los individuos de cara al trabajo. Para ello, dedicaremos un apartado inicial a analizar la terminología en la que se enclava el concepto de intraemprendedor, que, evidentemente, comparte raíz con el término matriz de emprendedor. Veremos en este apartado cómo es perfilado, revestido, el intraemprendedor en la literatura empresarial, cuáles son sus cualidades, su retrato-robot en la comunicación empresarial $^{3}$. A continuación, en un segundo apartado, más allá de los aspectos terminológicos y de la literatura managerial, abordaremos la contextualización de cambios en el trabajo y en las empresas en estas últimas décadas que han condicionado el ascenso

Metodológicamente, esta reconstrucción del perfil del emprendedor se ha realizado mediante la consulta de noticias de prensa, blogs, artículos en revistas mensuales de gestión y en revistas especializadas académicas del área de economía y empresa. La búsqueda del término “intraemprendedor” en la base de datos de prensa Factiva ofrece 177 resultados en prensa diaria o semanarios. E1 90\% de estas noticias están concentradas en los últimos 4 años. En 2013, comenzó a aparecer "intraemprendedor" como término especializado en el contexto de la ebullición de la familia terminológica emprendedora en esos años. Desde entonces hasta hoy, la idea de intraemprendedor ha visto aumentar sus apariciones. En cuanto a las revistas académicas especializadas en empresa, la búsqueda del término inglés intrapreneur en la base de datos IDEAS ofrece 41 artículos (con 19 apariciones en abstract). La búsqueda daría un mayor número de resultados de ampliarse los términos como corporate entrepreneurship o entreployee, entre otros, y de consultarse otras bases de datos bibliográficos, pero dado el carácter exploratorio de este artículo no se ha considerado intensificar la búsqueda de estas referencias. Por su parte, Dialnet ofrece 31 referencias en castellano en el área de ciencias de la empresa. En cuanto a las revistas mensuales, se han realizado búsquedas en Capital Humano (Wolters Kluwer) y Emprendedores. Por su parte, los blogs y los artículos de divulgación en publicaciones digitales ligadas a asociaciones, instituciones de enseñanza o empresas viven una efervescencia en torno a la idea de emprendedor y de intraemprendedor. La reiteración de contenidos y la superficialidad en el tratamiento de la cuestión por parte de estas fuentes no resta, sin embargo, interés a este florecimiento del concepto de intraemprendedor y se han utilizado para completar el análisis. En estos medios más divulgativos, son numerosos los textos basados en historias de éxito empresarial, donde hombres de empresa de mayor o menor éxito, dan testimonio y relatan su experiencia. Este género se ha extendido mucho en el mundo empresarial, que se aleja tantas veces de los formatos y contenidos más académicos y/o especulativos para volcarse en la narración viva y pragmática de una experiencia empresarial. Estos retratos, a la manera de una galería de celebridades, exponen apologéticamente la fe empresarial y ofrecen una guía paso a paso del éxito que da confianza a sus seguidores. Este es el secreto de sus enormes ventas cuando se publican en forma de libro.

Una selección de todos los anteriores materiales ha servido para realizar un análisis temático en torno a la definición, los perfiles y los rasgos característicos del intraemprendedor que se recoge en el primer apartado de este artículo. 
del intraemprendedor. El posfordismo ha sido un vivero de transformaciones en la organización del trabajo que apelan a la idea del intraemprendedor. Recogeremos los análisis de algunos autores -entre otros muchos posibles- que desde las ciencias sociales han dedicado una atención particular a esta idea del trabajador emprendedor. Un tercer capítulo de conclusiones servirá de cierre a este artículo.

\section{El surgimiento del intraemprendedor en la retórica empresarial}

\subsection{Aspectos terminológicos previos de la noción de intraemprendedor}

La moderna retórica empresarial que ensalza el cambio permanente no podía dejar de ser también cambiante en la forma de nombrar y usar la terminología para describir el fenómeno emprendedor. A la cultura innovadora de la empresa le acompaña una continua innovación en el léxico y la terminología con los que nombra sus procesos. Este ha sido el caso del término emprendedor. Sintagma exitoso, sobre todo durante esta última década, que condensa las virtudes de la conducta empresarial y a la vez la renueva. En los momentos de máxima efervescencia del término en España (en torno a 2010 se duplicó el número de noticias que contenían la palabra emprendedor), el término emprendedor y el acto de emprender convivieron con otras variadas denominaciones -"emprendedurismo", "emprendizaje", "emprendurismo"- (Santos, 2014). Finalmente, parece que esta profusa y acelerada dinámica terminológica se ha frenado con las denominaciones de origen "emprender" y "emprendedor", que se han consolidado hoy con su inclusión en la última edición del Diccionario de la Real Academia de la Lengua.

El hecho de contar con un término más estable como es el de emprendedor no ha frenado la proliferación de denominaciones que le acompañan y que nos describen sus múltiples declinaciones, modalidades y matices. Las traducciones de la abigarrada literatura empresarial anglosajona han incorporado al léxico hispánico otras variedades en la taxonomía del campo emprendedor. Así, puede destacarse una especie particularmente valorada como es la del "emprendedor en serie", que no se conforma con emprender una sola vez, o la del "reemprendedor", que se hace cargo de una empresa cedida o no exitosa. Precisamente, el posible fracaso de la empresa es una ocasión para intentarlo de nuevo y esto constituye una de las esencias que se elogia repetidamente en el ecosistema emprendedor. El "emprendedor social" es otra variante destacada, que incorpora el adjetivo social al complejo universo emprendedor, en el que se trata de ajustar el difícil encaje entre el lucro y el servicio social. La proyección social del emprendedor es uno de los tópicos más difundidos en estos últimos años. Ello obliga aquí a hacer mención al acento filantrópico y preocupación por las causas justas que reposa en el ADN emprendedor y que ha dado lugar a toda una discusión encuadrada en el filantrocapitalismo, practicado sobre todo por la aristocracia emprendedora, que ha reactualizado las formas de caridad y el filantro-marketing, que se ha convertido en una especialidad en la formación de los directivos (McGoey, 2014; Duvoux, 2015).

De más reciente aparición, y menos conocido, es el término "intraemprendedor", que aquí nos ocupa, y que describe al trabajador que dentro de una empresa tiene 
comportamientos emprendedores o crea incluso su propia empresa. Esta especie del intraemprendedor, recién llegada al repertorio terminológico de la cultura emprendedora, está destinada a extenderse dada la consabida capacidad colonizadora y afán de totalidad que caracterizan a la difusión del espíritu emprendedor. Con la buena intención de resolver dudas lingüísticas, la Fundación del Español Urgente (Fundéu), asesorada por la Real Academia, ha propuesto algunas alternativas para evitar el neologismo que supone el uso de "intraemprendedor" y sugiere sustituirlo por "emprendedor interno", "empleado con iniciativa", "trabajador creativo", "persona con dinamismo", "empleado proactivo". Nuevos vocablos que, sin embargo, agregan matices particulares y complican la clasificación intraemprendedora. En todo caso, la difusión del término intraemprendedor se mueve aún en círculos especializados y no ha aparecido con fuerza en los medios de comunicación. Ello no quita que la extensión del carácter emprendedor a los propios trabajadores sea ya un sólido indicio de la presencia de los discursos empresariales en cualquier ámbito de la realidad laboral y social.

En este particular bestiario del mundo emprendedor, han aparecido también, afortunadamente, lecturas críticas que, desde el campo académico de las ciencias sociales y humanas, han puesto el acento en las fallas del modelo de emprendedor y contrastan abiertamente con el optimismo que este desprende en las lecturas manageriales. Esta crítica contraemprendedora opera alterando la grafía de la palabra "emprendedor", consiguiendo denominaciones ocurrentes y de gran poder expresivo. Sus orígenes son, a veces, difíciles de encontrar, pues en muchos casos provienen de la crítica popular. Frente al emprendedor, encontramos, así, nuevas variantes como el "Emperdedor" (Brieva, 2015), que ha encontrado una replica en la televisión con el reportaje de Jordi Évole "\#emperdedores". Asímismo, encontramos el "Emprendeudor" (Moruno, 2015; Briales, 2017); el "Lumpenemprendedor" (Santos, 2014) o el "INEMprendedor" (Serrano, 2016), una variedad particularmente perversa con cuerpo de parado y cabeza de emprendedor, que vuelve a demostrar los usos activadores que contiene el discurso emprendedor aplicado, en este caso, al colectivo de desempleados. La cercanía fonética ha llevado a la literatura más radical a jugar con el binomio "emprendedor/depredador".

Esta crítica contraemprendedora no ha pasado desapercibida en otros países, donde entre los especialistas de la cuestión podemos descubrir el "self-emprendedor" (Bröckling, 2015), el autoentrepreneur (Abdelnour, 2017), el anthropreneur (Linhart, 2015) o el entreprecariat (Lorusso, 2016). Este último vincula los actuales destinos emprendedores que esperan al precariado cognitivo en su errante recorrido laboral, que tiene como punto de llegada el freelancismo o el trabajo independiente, lo que pone sobre la mesa la creciente presión empresarial al conjunto del precariado. Más ajustado al objeto de este artículo, descubrimos el término entreployee (Pongratz y Voß, 2003), que sugiere una fusión entre el empleado y el emprendedor. El término entreployee proviene del emprendedor y gurú de la autoayuda James Altucher (2013) que en su libro de elocuente título -Choose yourself- propone elegirse a uno mismo como jefe dentro de la empresa.

Esta ingeniosa marmita de neologismos en torno al emprendedor está destinada a ampliarse pues abre toda una combinatoria crítica de nuevas prácticas y conceptos vecinos al de emprendedor. No sería extraño ver aparecer, por ejemplo, la "emprendibilidad", donde se fusionaría la empleabilidad con el espíritu emprendedor; el "ubertariado", que traería el cruce del proletariado con el moderno capitalismo de 
plataforma -hijo predilecto del emprendimiento- o, siguiendo la misma inspiración, el "prolestartupriado". Estas amalgamas, a veces imposibles, cobran vida en el contexto de acoso textual de estos últimos años en que la reiteración de la palabra emprendimiento en todos los medios se ha infiltrado hasta en nuestros espíritus gracias al "espíritu emprendedor", que anuncia que la empresa siempre va contigo.

Señala el escritor Juanjo Millás, haciéndose eco de las ideas de algunas corrientes del psicoanálisis, que los humanos somos un instrumento del lenguaje, no sus dueños, somos colonizados por él. El niño no aprende a hablar, es paulatinamente colonizado por el lenguaje. Sin entrar en la discusión teórica que hay detrás, es cierto que esta colonización de las prácticas y los discursos por parte de la retórica empresarial es un hecho cada vez más indiscutible en la realidad social actual. Todos estos términos no agotan la lista de denominaciones en esta prolija retórica empresarial (Alonso y Fernández Rodríguez, 2013). Es, por tanto, necesario seguir su pista ya que en los discursos emprendedores, empresariales, de hoy encontramos rasgos de la precariedad que cobrará forma mañana.

\subsection{El intraemprendedor como arquetipo del trabajador emprendedor e innovador en la literatura de gestión empresarial}

Los especialistas en gestión y recursos humanos han insistido a partir de la década de 1990 en la transición de un modelo de empleo fijo hacia un modelo de trabajador emprendedor. Este último garantizaría la flexibilidad requerida por las nuevas formas de organización del trabajo que se imponen en los sistemas postfordistas. Desde entonces, la autonomía, la responsabilidad son las nuevas competencias que los asalariados tienen que aportar y la empresa promover. Desde los momentos iniciales en que se establece el origen del término intrapreneur (Pinchot, 1985; Cadar y Badulescu, 2015), sus partidarios en el campo de la gestión de empresas propugnan una transformación integral en la empresa. No solo ha de reorientarse la concepción del trabajador para ser entendido como un empleado que aporta una innovación o desarrolla una idea de negocio dentro de la organización, sino que esta ha de orientarse, asimismo, hacia el corporate entrepreneurship, potenciando el comportamiento emprendedor a todos los niveles y creando las condiciones para que los trabajadores emprendan en el seno de la empresa. Ello conduciría directamente a la mejora de la rentabilidad de la organización, a una mejora de la posición competitiva o a la renovación estratégica de un negocio ya existente. Un modelo, por lo tanto, donde la concepción empresarial alcanza a todos los empleados y donde cada parte que integra la empresa es de hecho una empresa. En el debate posterior de las ciencias de gestión empresarial a partir de la década de 1990, el corporate entrepreneurship ha incluido dos tipos de enfoques complementarios: por una parte, el nacimiento de nuevas empresas en el seno de empresas ya existentes impulsadas por intraemprendedores internos y, por otra parte, la transformación de las organizaciones para hacer esto posible en pro de la innovación (Hernandez, 2006: 19). En esta misma década, en el campo académico de la gestión de empresas, la idea de corporate entrepreneurship ha sido una de las vías de crítica de las empresas "piramidales" y "mastodónticas" características de periodos anteriores y dominadas por un funcionamiento burocrático e ineficaz. La nueva gestión propone reorganizar los organigramas para transformar las empresas en redes empresariales compuestas por pequeñas entidades 
autónomas dirigidas como pequeñas empresas. El necesario equilibrio en la convivencia de los intraemprendedores en el seno de la empresa madre ha dado lugar en la literatura de gestión a toda una línea de investigación para que los primeros no acaben con la empresa que les permitió desarrollar su proyecto de empresa interno. Se alude a empresas que promocionaron esta vía sin las cautelas necesarias y que tuvieron un resultado catastrófico como Enron (Hernandez, 2006).

Sin embargo, entre los expertos predominan los puntos de vista positivos sobre la estrategia intraemprendedora siempre que se examine con detalle cómo llevar adelante el procedimiento. Entre los estudios que identifican las buenas prácticas para poner en marcha procesos de intraemprendedurismo se destacan las siguientes (Bouchard y Fayolle, 2011): compromisos firmes de la dirección, desde las declaraciones hasta la puesta en marcha de medios concretos; creación de espacios de autonomía, en estos espacios los empleados encontrarán libertad de acción y medios para realizar sus proyectos innovadores o divergentes. Esto podría conseguirse creando dentro de la empresa una entidad independiente del conjunto de la empresa matriz, dotada de recursos propios y sin vínculos con otras divisiones operativas. Podría conseguirse, igualmente, creando recorridos orientados dentro de la empresa para empleados capaces de llevar adelante un plan de nuevo negocio sin necesidad de depender de su superior directo. Podría conseguirse, finalmente, a través de la creación de unidades operacionales descentralizadas dentro de la empresa. Dentro de las buenas prácticas se destaca la evaluación y el seguimiento de estas iniciativas; la intensificación de la comunicación en el seno de la empresa; la puesta en marcha de un sistema de incentivos, sea a través de primas salariales, de participación en la rentabilidad de la nueva empresa creada o en su propiedad. Junto a estos aspectos, se destaca la necesaria política de formación y sensibilización de los trabajadores $\mathrm{y}$, finalmente, una gestión del tiempo de trabajo favorecedora del intraemprendedurismo. Este es uno de los aspectos más divulgados. Algunos de los casos más exitosos de empresas intraemprendedoras se caracterizan por ceder un porcentaje del tiempo de trabajo para que los trabajadores intraemprendan, como $3 \mathrm{M}$-donde podrían dedicar un 15\%- o Google, que puso en marcha sistemas donde el "quinto día" de la semana podría destinarse al autoemprendimiento. Como no podría ser de otra manera, las formas operativas de las empresas para llevar a cabo la organización del intraemprendimiento y la terminología que los expertos crean para ello son abundantes ("célula intraemprendedora", "bucle intraemprendedor", "cabeza de puente intraemprendedora" o "plataforma intraemprendedora") y presentan variedades de acuerdo al tamaño y los medios o las relaciones dentro de la empresa (Bouchard y Fayolle, 2011). En todo caso, sea cual sea la estrategia adoptada, se identifica a las empresas emprendedoras como un ejemplo de innovación gracias a que desarrollan el talento interno y lo ponen al servicio de la empresa, que se convierte a su vez en una incubadora de empresas. Como los propios especialistas ponen de manifiesto (Lisein y Degré, 2011), esta es una estrategia de innovación crucial para las empresas sea cual sea la manera de desarrollarla: a través de aprovechar al intraemprendedor espontáneo; poner recursos a su disposición; planificar el intraemprendimiento a través de planes de desarrollo de proyectos concretos basados en ideas de negocio o formalizarlo en estructuras definidas en el organigrama de la empresa. 


\section{3.-El intraemprendedor y sus metáforas: perfiles y rasgos de carácter}

Además de los anteriores aspectos organizativos dedicados a la empresa emprendedora, resulta interesante profundizar en el arquetipo del intraemprendedor tal y como queda registrado en el discurso del nuevo management. La denominación de intrapreneur no se libra de la volubilidad que aqueja a la terminología en los medios de negocios y en la literatura de gestión (desde las secciones de economía y negocios en prensa diaria hasta artículos en revistas especializadas). Así, se ha podido distinguir al innerpreneur, que pone el acento de la creación de empresas más en la dimensión de su desarrollo personal que en la creación de negocios y se asocia con los trabajos creativos o también al intrastartup, que especifica la actividad intraemprendedora en una empresa startup. Pese a todo, la denominación en inglés de intrapreneur conserva una considerable estabilidad desde su surgimiento a mitad de la década de 1980 -incluso con menciones anteriores- y es la que se ha divulgado posteriormente a otros idiomas, como es nuestro caso con el intraemprendedor, que además permite incorporar la impactante semántica reciente en España del término emprendedor.

Más importante que el propio origen y la variedad terminológica del intrapreneur, resultan las metáforas que se le asocian en los diferentes documentos consultados. Las más extendidas identifican al intraemprendedor como un "soñador" que construye empresas, un "héroe", un "creador", un "motor de progreso", el "arma secreta" de la empresa, un "trabajador aumentado", en referencia a la realidad aumentada con que las nuevas tecnologías redimensionan la realidad, "el empleado perfecto". No cabe duda del idilio entre la empresa actual y el trabajador intraemprendedor. En el modelo ideal de este, se destaca su carácter revolucionario, es un "transgresor del orden", incluso se le compara con un "desviado" pues introduce innovaciones rupturistas con el antiguo régimen empresarial. En esta misma línea, se establece el símil con el viaje: es un "cosmopolita", un "traductor", "alguien que vuela por encima de los demás", un "pionero". Cada época vive su fiebre del oro que en nuestro momento contemporáneo parece protagonizar esta mentalidad emprendedora.

El perfil con que es descrito configura un modelo ideal en la línea con los símiles anteriores. En el decálogo que aparece reiteradamente en las revistas, blogs y artículos, el intraemprendedor es curioso, abierto al cambio, experimentador, apasionado, rebelde, no teme equivocarse, rompe rutinas, no puede vivir sin proyectos para gestionar (Mack, 2001; Alter, 2011).

El análisis de 100 casos de intraemprendedores ha llevado a Louis Jacques Filion (2016) a construir un retrato robot del intraemprendedor compuesto por ocho rasgos principales: el compromiso, la curiosidad, la imaginación, la conquista (capacidad para apropiarse de algo que no estaba definido previamente), capacidad de negociación (para desarrollar sus proyectos no solo debe obtener recursos, sino también poner de acuerdo a actores de su empresa con intereses diferentes), encontrar aliados, pragmatismo y resultados y, finalmente, capacidad de aprender. Un retrato robot que podría completarse con otras variables personales y empresariales recogidas en diferentes estudios realizados a escala internacional (Bosma, Stam y Wennekers, 2012; Douglas y Fitzsimmons, 2013).

En la literatura más propagandista de la cultura intraemprendedora, difundida en decenas de webs, blogs, folletos y otros medios, se identifica al intraemprendedor con un "apasionado", no es un trabajo, sino una "forma de vida" "idealista", "rebelde", "soñadora". Esta forma de vida se presenta rodeada de una épica del esfuerzo, 
donde se combinan "momentos de pasión y euforia, pero también largas esperas y soledad en hoteles a miles de kilómetros de su casa, lejos de su familia y amigos". El intraemprendedor está particularmente dotado para moverse en "lo desconocido", en la "aventura apasionante", pues "lo auténtico está más allá de donde busca la masa". Este elitismo se acompaña de un signo de identidad distintivo: "la mentalidad ganadora" donde se trata de competir, ganar, y, si hace falta "machacar" al rival. En Intraemprendedores. Reinventa tu empresa con espiritu start-up, Vega (2016) enmarca la figura del intraemprendedor en el espacio prominente de las startups, para las cuales no hay mejor manera de innovar que a través del intraemprendedor. Es decir, creando desde la startup la propia competencia interna para generar nuevos negocios. En este contexto startup, se plantea el colmo del emprendedor: el fundador de una startup que se reemplaza a sí mismo en la dirección de la empresa y cede la batuta a un director ejecutivo, con más capacidad para la gestión, para que lo reemplace, pasando el fundador a convertirse en intraemprendedor dentro de la empresa. Esta figura se perfila así como una garantía para la reinvención de las empresas más innovadoras.

\section{La figura del intraemprendedor en el núcleo de los cambios del posfordismo. Una revisión desde las ciencias sociales}

El arquetipo del trabajador implicado, innovador, participativo es constante en la modernización de los procesos de organización del trabajo a lo largo del posfordismo. En el apartado anterior, hemos descrito cómo este hecho se refleja en los textos de ciencias empresariales. En este segundo epígrafe, nos centraremos en los análisis realizados sobre esta cuestión desde las ciencias sociales y humanas. Son muchos los autores que han abordado desde la sociología, la economía y la filosofía la reconceptualización del trabajador y pensamos que su revisión puede aportar dimensiones interesantes a la genealogía del intraemprendedor.

Comenzamos por las interpretaciones que realizan al respecto algunos autores cercanos al concepto de capitalismo cognitivo, donde puede enclavarse la figura del intraemprendedor como uno de los resultados evidentes de las nuevas formas de acumulación del capital en el posfordismo. Aunque el término intraemprendedor no se enuncia explícitamente en la teoría desarrollada por el capitalismo cognitivo, de sus argumentaciones cabría extraer que esta figura emerge como prototipo ideal de trabajador, como pieza deseada de la nueva organización del trabajo en el ciclo cognitivo de la acumulación capitalista. En la lectura de los principales autores de esta corriente (Marazzi, 2003; Vercellone, 2009; Fumagalli, 2010), estos procesos se van concretando a lo largo de la década de 1990, conforme cobran forma los rasgos centrales del capitalismo cognitivo. En primer lugar, por la creciente importancia del conocimiento y su necesario control de cara al proceso de acumulación y valorización del capital y, en segundo lugar, por la acentuación de la globalización como escenario de la organización del trabajo. Estos procesos generan nuevas economías en la producción de saber, conocimiento y aprendizaje y en su control y apropiación capitalista. La producción de este saber por parte de los trabajadores trae a primer plano la necesidad de las empresas de controlar las facultades vitales y cognitivas de estos. Ciertamente, ello provoca cambios tanto en la naturaleza del trabajo como 
en las fuentes de valorización y en la estructura de la propiedad. Entre los teóricos del capitalismo cognitivo, la importancia de las facultades vitales en la producción del saber ha llevado a hablar de bioeconomía, biocapitalismo cognitivo (Fumagalli, 2010). Una de las tesis centrales de esta corriente es la progresiva importancia del trabajo inmaterial en la creación de valor. Este valor se basa, así, en las capacidades humanas cognitivas, relacionales, emocionales, lingüísticas. La compleja composición del trabajo inmaterial excede el ámbito de las instalaciones de trabajo, puede originarse en redes externas a la empresa y resulta difícil de distinguir el tiempo de trabajo y el tiempo de vida. A raíz de este carácter decisivo de la dimensión cognitiva del factor trabajo en la generación de valor, la pregunta que se plantea es cómo el mundo empresarial puede captarlo y controlarlo. Este interrogante sobre las nuevas formas de subordinación, de explotación, de subsunción es el que guía el análisis de los teóricos del capitalismo cognitivo.

En este sentido, hay que destacar la singularidad con que se expresa el trabajo cognitivo-relacional en términos de las capacidades individuales lingüísticas, emocionales, cognitivas de cada empleado, lo que se traduce en una gran dificultad para homogeneizar y organizar el trabajo. Christian Marazzi (2003) subraya cómo en el cambio de los procesos de acumulación, los factores de crecimiento necesarios responden a un modelo antropogenético, son preponderantemente imputables a la actividad humana, a los aspectos cognitivos, comunicativos, relacionales, de ideación innovadora y creativa. El control de estos factores puede escapar a las empresas, ya que no controlan del todo ni su formación ni su ejecución. Ello impone necesariamente nuevas formas de ejercer dicho control. Frente a las formas de subsunción formal y real que caracterizaron la explotación en el fordismo, los cognitivistas han estudiado las nuevas formas de subsunción vital que se expanden en el posfordismo. Resumidamente, hay que señalar que esta se consigue, en primer lugar, a través de una nueva intensificación del trabajo consistente en el debilitamiento de las fronteras entre tiempo de trabajo y tiempo de vida, lo que permite ampliar los marcos de acumulación, permite poner la vida a trabajar. En segundo lugar, se consigue a través de la progresiva fusión del individuo trabajador en la empresa, en este caso lo que se debilita es la frontera entre la identidad empresa y la identidad de trabajador. Lo que pasa a ser propiedad de la empresa no es únicamente un bloque de tiempo, sino que se trata de acceder a las capacidades cerebrales. El cerebro se convierte en máquina productiva. Estos dos procesos son determinantes en la idea de intraemprendedor, que resultaría el arquetipo perfecto de la nueva subsunción vital del trabajador-empresa en el marco de las nuevas formas de acumulación del capital.

Además de este dominio sobre los trabajadores, la empresa necesita ir más allá en el control de los procesos de creación de conocimiento. La importancia estratégica de este en el nuevo modelo de acumulación conduce al capital no solo a usarlo y aplicarlo para organizar el trabajo, sino a apropiárselo para dominar y controlar. El control tecnológico y, sobre todo, la apropiación empresarial de los significantes referidos a la innovación, creatividad, talento o formación presentan el conocimiento como un producto de la empresa, lo sitúan en el campo semántico empresarial. Así como la fábrica fue legitimada como espacio del progreso o del crecimiento económico a lo largo de la industrialización, hoy se presenta, además, como el lugar de la innovación. Esta nueva legitimación deshabilita o dificulta la crítica a la acción de la empresa, facilita el control del trabajo y consolida su lugar de dominio. 
Por añadidura, el control del trabajo en la empresa postfordista se consigue mediante la creación de imaginarios individualistas a través de una exacerbación del mérito, la competitividad y la relectura individualizadora del capital humano como dispositivos de gobierno de los individuos. Esta lucha competitiva de los individuos y de puesta a prueba de sus capacidades productivas, de su motivación, es regulada y medida por los sistemas de evaluación, de calidad, los mecanismos de emulación, las distinciones y el acceso diferencial a recursos. En sus versiones más recientes, se reclama la conversión del individuo en marca personal -personal brand-, lo que extrema la individualización competitiva y la diferenciación. La prestación propia queda marcada por la singularidad, la diferencia frente al otro. La idea de trabajador como marca glorifica la autonomía como competencia clave en el trabajo y en la profesión y se ensalza el trabajo independiente y la figura del emprendedor. Por el contrario, se sospecha del trabajo dependiente en cuanto a posible freno de la iniciativa y del potencial personal. Paradójicamente, una vez convertidos en marcas singulares y diferenciadas, todos quedan homogeneizados por el hecho esencial de ser marca, por la marca como norma. En resumen, las nuevas formas de control basadas en la identificación del trabajador con la empresa, en la autonomía, en la difuminación de las fronteras trabajo/vida y en la exacerbación de los imaginarios individualistas son el caldo de cultivo para la divulgación de figuras intraemprededoras.

En sintonía con las interpretaciones expuestas por parte de los teóricos del capitalismo cognitivo, los sociólogos alemanes Hans Pongratz y Günter Voß (2003) analizan específicamente la figura del entreployee como fruto de cambios en los procesos de organización del trabajo en el posfordismo. El entreployee -una fusión de los términos entrepreneur y employee- es, de acuerdo con Pontgraz y Voß, el resultado de las estrategias empresariales que promueven dichos cambios. El modelo de employee fordista se deja atrás para proponer el nuevo arquetipo de entreployee que responda a las necesidades impuestas por las mutaciones en las formas de trabajo: flexibilidad, autonomía, participación y cooperación, a las que añaden otras como el teletrabajo, la externalización y la creciente utilización de trabajo independiente por parte de las empresas (sobre todo en sectores ligados al conocimiento). En los procesos de control del trabajo, el entreployee introduce un cambio que lleva del control externo de la mano de obra, de la vigilancia externa, al autocontrol, a la promoción activa de la autorresponsabilidad en los empleados.

Aunque los sociólogos alemanes no entran a fondo en la dimensión subjetiva del entreployee y se centran más en las dimensiones materiales del cambio laboral, adelantan la conjetura de que el entreployee podría convertirse a largo plazo en un modelo normativo que difunde un sujeto empresario. Ponen para ello los ejemplos de estilos de gestión de recursos humanos basados en el lema "Selbst GmbH" "Yo Ltd. Co.") o los programas de reforma del mercado de trabajo del gobierno alemán emprendidos por la Comisión Hartz, al inicio de la década de 2000, bajo la idea del yo-empresa -"Ich $A G$ " ("Yo Inc."). En buena medida, las evoluciones posteriores han dado la razón a las conjeturas de Pontgraz y Voß muy aproximadas a la temática que nos preocupa en este artículo sobre la idea de intraemprendedor. Los sociólogos alemanes conjeturaron que esta nueva categoría del entreployee podría traer consigo cambios profundos en la naturaleza del empleo y en la organización del trabajo, como por ejemplo problemas de salud laboral asociados a la incertidumbre, a los procesos de individualización o a otras inseguridades ligadas al trabajo freelance y a 
lo que ellos denominan "jornaleros autoemprendedores" que abrirían nuevas formas de mercantilización de la fuerza de trabajo.

Por abundar en otros autores de referencia, la publicación de El nuevo espíritu del capitalismo de Luc Boltanski y Ève Chiapello (2002) ofrece un fresco completo sobre los mismos temas. Concretamente en su reconstrucción de la cité par projets, los sociólogos franceses plasman la imagen del trabajador emprendedor apoyándose en una metodología basada en el análisis de la literatura empresarial. Esta reconstrucción de la "ciudad por proyectos", tal y como emerge de esta literatura, recoge los grandes tópicos que configuran la idea de este nuevo tipo de trabajador demandado para orientarse en los nuevos entornos cambiantes del trabajo: un trabajador "entusiasta", "adaptable", "flexible", "disponible", "digno de confianza", "autónomo", "empleable", "polivalente", "dispuesto al riesgo", entre otros muchos adjetivos recogidos en los textos analizados por Boltanski y Chiapello. De esos mismos textos, también emana la idea de que el mundo actual de la "ciudad por proyectos" es un mundo incierto, complejo, en movimiento, donde los trabajadores han de prepararse y valerse por sí mismos. El yo empresario es la única instancia que puede garantizar seguridad en ese contexto. El modelo tradicional de empleo asalariado estable deja de ser el criterio central de actividad -como lo era en la "ciudad industrial"- y se difuminan las fronteras entre trabajo asalariado y no asalariado, entre estabilidad e inestabilidad, entre lo mensurable y lo inconmensurable en el trabajo. De aquí la importancia de la autogestión de las propias competencias y capacidades. Esta es la mejor alternativa cuando la noción clave de proyecto se sitúa en el centro. La vida se entiende como una sucesión de proyectos en los que se participará de formas diferentes, lo que constituye una prueba de excelencia, en la que destaca una jerarquía de seres encabezada por los "jefes de proyecto", los "managers", gracias a su capacidad de "comunicación", de "mediación", de generar "empleabilidad", de "tejer redes" y de "innovación" (ibíd., 163-173). La "ciudad por proyectos" merecería sin duda un mayor tratamiento en este artículo por su interés en sí y por el desglose de temas que recoge de cara al perfil emprendedor del trabajo, pero incluso con la limitada atención que se le ha podido prestar aquí, es indudable la consonancia de lo presentado con la idea de intraemprendedor.

Por su parte, en el análisis de las derivas postfordistas que estamos exponiendo, habría que añadir a André Gorz $(2001,2003)$ quien reflexiona, a finales del XX, sobre las estrategias patronales para "desplazarse desde una dominación directa de la actividad de los trabajadores hacia una dominación basada en la producción de sí" (2001: 63). Este tránsito puede también seguirse en el auge del modelo de cualificación por "competencias" -entendidas como "talentos de improvisación", innovación e invención continua- frente a los viejos "saberes". O, Igualmente, en el cuidado y la producción del propio capital humano por parte del trabajador, capital que es captado después por la empresa. O en la utilización de los grupos de trabajo autónomos como forma de organización del trabajo, que ofrecen una imagen de la gran empresa como "constituida por pequeños emprendedores autónomos" debido a la "trasferencia de competencias empresariales hacia la base" (ibíd., 61). Las propias declaraciones del empresariado que Gorz cita expresan claramente el sentido de estos cambios cuando consideran a los empleados -"colaboradores"- como parte del capital de la empresa. Nuevamente, el intraemprendedor se alza como figura emergente.

La lista de autores tratados en este apartado se completa con Pierre-Michel Menger (2003) y su tesis acerca de cómo el trabajo artístico se está convirtiendo en 
el modelo paradigmático de trabajo. Esta idea aporta dimensiones interesantes a la idea de intrapreneur. Menger considera que las evoluciones actuales del trabajo convergen con los rasgos típicos del trabajo artístico: implicación, autonomía, flexibilidad aceptada y reivindicada, mezcla de orígenes en la remuneración, uso estratégico del talento y la inventiva, aceptación de la movilidad, crítica de las jerarquías, motivación intrínseca y pasión, competitividad, capacidad para afrontar la incertidumbre y la inseguridad de las trayectorias laborales y gestionarlas económicamente. En el modelo de innovación empresarial actual resulta difícil identificar a priori lo que va a determinar el valor de lo producido. Esto produce una inflación de la significación de la originalidad, la inventiva, la singularidad o el talento, por otra parte, absolutamente arbitrario e indescifrable, además de generador hoy de desigualdades desmedidas.

Junto a las cualidades anteriores. Otro rasgo del trabajo artístico que lo aproxima a las evoluciones emprendedoras actuales es la extensión del trabajo independiente, freelance, muy difundido entre los artistas, trabajadores de la cultura, investigadores y otras profesiones de las industrias culturales. Esta condición de trabajo por cuenta propia posibilita una gran flexibilidad tanto en la contratación como en el cese de la relación laboral. El mercado de trabajo de estas profesiones presenta un gran excedente de mano de obra por cuenta propia que facilita la elección a las empresas y crea una competitividad que abarata la remuneración. Los propios trabajadores se hacen cargo de la incertidumbre que esta situación genera en términos de inseguridad, precariedad, multiactividad y subempleo. Las medidas de protección social para estos trabajadores y trabajadoras no están muy desarrolladas, por ello las salidas son individuales y la mejor garantía para no caer en el lado del riesgo es crearse una reputación. Con ella, siempre puede conseguirse el triunfo y siempre se puede adoptar el modo de trabajo freelance, trabajar por proyectos, innovar, tener más iniciativa, mejorar la formación y el aprendizaje permanente o asumir más riesgos.

Esta apoteosis del trabajo flexible que Menger identifica en el trabajo artístico se relaciona tanto con la actual hibridación de estatutos de empleo que difuminan las fronteras del régimen salarial como con las competencias emprendedoras del trabajador. Precisamente, estas competencias son la base de la diferenciación y la comparación de las capacidades individuales de los trabajadores $\mathrm{y}$, por tanto, representan los criterios de clasificación de los saberes, los recursos cognitivos, las disposiciones y el potencial que poseen. El "enriquecimiento" de las competencias deriva en un trabajador-emprendedor que "construye" su puesto de trabajo en vez de simplemente ocuparlo y construye su carrera fuera de los esquemas lineales típicos de las empresas fordistas. Al contrario, su capital de competencias se puede incrementar y transferir a otras empresas. Ser emprendedor de la propia carrera, como indica la literatura empresarial, significa construir un perfil personal y una reputación visible y reconocida más allá de los límites de una única empresa (ibíd. 83-84). Menger cierra su libro con un interrogante provocado por las incertidumbres que acompañan al modelo de trabajador artista: qué podemos hacer con la incertidumbre que genera la lógica del talento y la creatividad en un universo empresarial que necesita flexibilidad, pero que no asume la incertidumbre. Desgraciadamente, es aún hoy una pregunta que espera respuesta.

La idea de intraemprendedor, que fusiona el estatuto de trabajador y el de emprendedor, encaja en este marco de cambios en la organización del trabajo y en los procesos de subsunción que hemos relatado en este apartado que ahora cerramos. 
Como acabamos de ver en los textos de los autores tratados, la concepción intraemprendedora es al mismo tiempo una forma de organizar el trabajo y, asimismo, una forma de mentalidad y de gobierno en busca de modelar subjetividades. Nos hemos acercado a esta idea de subjetividad con las alusiones a los trabajos de Foucault y Deleuze (1999) sobre la sociedad de control, pero querríamos cerrar este apartado aludiendo a algunos de los actuales dispositivos de modelado (y automodelado) con que el régimen emprendedor (Bröckling, 2015) perfila esta subjetividad empresarializada a través de tecnologías sociales y del yo. Estas no se presentan necesariamente como una obligación para implantar un yo empresa, sino que constituyen un ethos, un conjunto de valores orientadores y de reglas de conducta que permiten al individuo evaluar (y autoevaluar) su existencia y ponerla al día conforme al ideal empresarial. La mayoría de los individuos se encuentran, no en el ser, sino en el llegar a ser emprendedor.

En este artículo, no se puede profundizar sobre toda la gama de estas tecnologías. Se han descrito muy adecuadamente en trabajos recientes (Laval y Dardot, 2013; Nicoli, 2015; Bröckling, 2015). Este último autor las analiza a través de cuatro conceptos clave que especifican diferentes facetas de la actual acción empresarial -creatividad, empoderamiento, calidad y proyecto-. Muchas de ellas han sido tratadas monográficamente, como es el caso de los mecanismos de evaluación y autoevaluación; de selección de personal; de orientación y formación profesional; de interiorización de la calidad; del rendimiento, la competitividad y la automedición; de los mecanismos de forja del liderazgo; de cultura empresarial e identidad corporativa (marca); de reorientación del conflicto; de la activación y el trabajo sobre las emociones -desde el mentoring, coaching y otras formas de desarrollo personal, hasta el uso de relatos movilizadores y de incitación a la implicación sobre la base del storytelling-. La idea de intraemprendedor es en sí un storytelling, cargado de metáforas, que se presenta como modelo ideal para la adaptación del sujeto en el trabajo. Todos estos mecanismos han servido tanto para subjetivizar la organización trabajo como para trabajar sobre la subjetividad de los empleados a la manera emprendedora. Por lo demás, es un proceso en marcha, inacabado.

Massimiliano Nicoli (2017) apunta la idea de "racionalidad start-up", "start-up existencial" para trazar algo así como la fase ulterior subjetivadora de la figura del emprendedor y toma como ejemplo la concepción de una de estas empresas, en eterno despegue, para establecer el símil con la vida y la carrera del trabajador contemporáneo. De hecho, las apelaciones a la reinvención continua, a aprender a considerar la propia vida como un work-in-progress, o a reponerse tras un fracaso muestran cómo el espíritu emprendedor no se construye de una vez, sino que es un trabajo de perfeccionamiento continuo que nunca acaba de completarse. Ello reclama una dedicación constante para devenir capital humano de primera calidad, adaptado a los continuos cambios en el mercado de las competencias. Reclama, también, aceptar la incertidumbre y los riesgos como algo inscrito en la propia existencia. La lógica más reciente startup no valora tanto al individuo calculador -que se ciñe a un plan de carrera-, sino que busca al experimentador del cambio continuo, que improvisa sobre el plan de empresa, lo que le acerca al perfil deseado de emprendedor/intraemprendedor innovador. Perfil que hemos visto anteriormente y que se acentúa en los best-sellers sobre startups más recientes.

En una línea concordante, el filósofo francés Michel Feher $(2007,2017)$ introduce nuevos argumentos sobre la subjetividad emprendedora poniendo al día las 
ideas de Foucault (2007) sobre el capital humano y actualizándolas con las recientes evoluciones del neoliberalismo que Foucault no vivió. Para Feher, el giro financiero del neoliberalismo ha traído cambios relevantes en la concepción del capital humano. No se trata ya únicamente de que el individuo acumule y maximice el stock de competencias que configuran dicho capital humano (a la manera de la concepción clásica de Gary Becker), sino que, acorde a la actual lógica financiera dominante, además se ha de mostrar un potencial, ha de mostrarse atractivo. No se trataría ya solo de aumentar la expectativa personal de una renta futura, sino de dar forma a una reputación, acreditarse, "apreciarse", mostrar que el valor de uno mismo seguirá aumentando, generar un impacto, en palabras de Feher, "apreciarse", ser un "gestor de una cartera de conductas" (Feher, 2014). En este sentido, moverse en el riesgo es una competencia añadida al modelo del emprendedor de sí. Lamentarse por la incertidumbre te aleja de este modelo de innovación.

Para Feher (2017), la financiarización impulsa la transición de un individualismo posesivo, que caracterizaba al homo economicus maximizador, a un individualismo especulativo, en el cual lo que cuenta es acertar en la apuesta de cómo construir la propia marca, la propia reputación, lo que se puede llegar a ser. Es justamente lo que se alaba en los modelos de empresa startup: la capacidad de atraer capital riesgo, demostrar potencial. Estas empresas ofrecen analogías para el comportamiento de los sujetos: gestores multitarea, en continua experimentación, que acumulan, miden y acreditan sus resultados en una secuencia sin fin de proyectos encadenados o simultáneos que metabolizan la incertidumbre. Del "empresario de sí" a la vida en modo startup.

\section{Conclusiones}

Tras el recorrido del artículo, son muchos los aspectos que hacen del intraemprendor una figura de relieve en el panorama de cambios laborales y empresariales actuales. A lo largo del periodo postfordista, la gran empresa se disuelve mediante la deslocalización, la externalización, la desregulación, la competición entre departamentos y, también, a través de políticas intraemprendedoras, que fomentan la creación de empresas por parte de trabajadores de la propia empresa. La empresa está dispuesta a ceder espacio a otra empresa -intraemprendedora- en su seno para captar el valor de la innovación y poder subsumir la creatividad del factor trabajo en el complejo marco del capitalismo cognitivo. Una empresa de empresarios en la que la lógica intraemprendedora torna la relación contractual en una relación empresarial entre empleado y empleador, lo cual modifica radicalmente el panorama de las relaciones laborales.

El intraemprendedor no solo transforma el marco de los procesos de trabajo y las relaciones laborales, sino que invoca la subjetividad de los trabajadores para acercarse a un ideal de conducta, a una forma de entender la vida. El intraemprendedor es un modelo ideal que apela al individuo como empresario de su fuerza de trabajo y de sus ideas. Hemos repasado en la primera parte de este artículo todo el repertorio de consejos movilizadores con que la retórica emprendedora pretende alfabetizar a los individuos en el espíritu emprendedor. Una retórica con espíritu misionero, indoblegable, omnipresente, expresada con autosatisfacción y autoalabanza, que busca constituir una comunidad emprendedora. 
Hoy es difícil saber hasta qué punto este espíritu emprendedor ha cuajado en la subjetividad de las personas en general y de los trabajadores en particular. Aparentemente, la incidencia es intensa, pero acotada a los segmentos más cualificados de trabajadores del conocimiento. A tenor de los datos que nos pueden ayudar a medir la difusión del espíritu emprendedor o intraemprendedor, los resultados no son tan prometedores para el nuevo espíritu del capitalismo informacional. Al contrario, los datos del estudio State of the Global Workplace 2011-2012 de la empresa Gallup (Crabtree, 2013) muestran que solo el 13\% de los empleados de todo el mundo participan o se sienten implicados en su trabajo, se muestran "psicológicamente comprometidos y con capacidad para hacer contribuciones positivas en sus empresas". Un 63\% se manifiestan no implicados, "carecen de motivación y es menos probable que inviertan esfuerzos discrecionales en los objetivos o resultados de la organización". Finalmente, un $13 \%$ se encuentran activamente desmotivados, lo que, en palabras del citado informe, "indica que son infelices e improductivos en el trabajo y propensos a transmitir negatividad a los compañeros". Los datos varían por área geográfica. En Estados Unidos, el porcentaje de implicados sube hasta el $29 \%$, pero en la vieja Europa, este grupo cae hasta el 14\%. Este mismo porcentaje se da en España.

En consonancia con los datos anteriores, las cifras sobre la situación de los intraemprendedores en España no son muy optimistas. En fechas recientes, el Centro Internacional Santander Emprendimiento (CISE) (2017) ha presentado el informe Emprendimiento corporativo en España. Gacelas y elefantes bailan sin pisarse en el que se analiza la realidad intraemprendedora y los apoyos que encuentra en las principales empresas españolas. El informe utiliza datos del Global Entrepreneurship Monitor (edición 2015), donde se mide la tasa de emprendimiento corporativo, que se define como el porcentaje de la población entrevistada de 18-64 años que, en los últimos 3 años, ha liderado una iniciativa emprendedora para la organización en la que trabaja (nuevo producto/servicio, nueva empresa, nueva unidad de negocio, etc.). Esta tasa se sitúa en el 1,8\% de la población entrevistada en 2014. El nivel es muy bajo y posiciona a España en el furgón de cola de los principales países ricos. Sin embargo, el mismo informe muestra unas expectativas desbordantes cuando la muestra considerada recoge las opiniones de las empresas. En este caso, un $86 \%$ de las 35 grandes empresas consultadas ha puesto en marcha programas para promover el intraemprendimiento y de estos programas, el $76 \%$ han comenzado a partir de 2011. Es por tanto un fenómeno en auge. E1 91\% de las empresas cree que la actividad intraemprendedora está destinada a aumentar en un futuro inmediato. Por ahora,

\footnotetext{
El sondeo utiliza los datos de la encuesta mundial Gallup realizada a una muestra de 73.752 trabajadores mayores de 18 años encuestados en 141 países, muestra que se completa con los 151.335 encuestados en Estados Unidos. Para medir la implicación en el trabajo, el sondeo incorpora al cuestionario la batería de preguntas de su Gallup Q12, un cuestionario diseñado para medir la implicación en el trabajo compuesto por ítems como los siguientes: ¿A mi supervisor, o alguien del trabajo, parezco importarle como persona? ¿Hay alguien en el trabajo que fomente mi desarrollo? ¿Mis opiniones cuentan en el trabajo? $O$ ¿Durante este último año, tuve la oportunidad de trabajar para aprender y crecer?

Gallup Q12 se basa en la idea de que la implicación en el trabajo hace crecer la productividad y el clima laboral de la empresa y lleva adelante toda una métrica que se ofrece a las empresas para mejorar sus resultados. También hay datos basados en Q12 para los directivos en el informe State of the American Manager: Analytics and Advice for Leaders. Los resultados de estos niveles directivos vuelven a ser desalentadores en cuanto a su imagen intraemprendedora: un 35\% de los directivos se sienten implicados frente a un 51\% no implicados y un $14 \%$ activamente no implicados.
} 
se observa una fuerte divergencia entre los discursos optimistas de las empresas y las prácticas de los intraemprendedores representadas por las bajas tasas.

Los datos anteriores muestran, por un lado, la escasa implicación de los trabajadores, que responde probablemente al poco atractivo de sus trabajos reales y, por otro lado, el énfasis de las empresas en dar publicidad al discurso emprendedor. William Davies (2016) dedica todo un capítulo de su libro La industria de la felicidad a describir la epidemia de aburrimiento en el trabajo y de enfermedades laborales que provienen de la falta de entusiasmo en el trabajo. Esto supone un obstáculo para la viabilidad del capitalismo a largo plazo. La preocupación se extiende entre la elite empresarial que trata con sus discursos actuales de humanizar e inyectar pasión emprendedora en el mundo laboral.

Sin ahondar en las tendencias de largo plazo, en el momento actual, a corto plazo, la difusión de las prácticas emprendedoras, allí donde han cuajado más, abren una agenda sociológica de temas que reclaman atención inminente. Por señalar dos de ellos brevemente, destacaríamos, en primer lugar, la inquietante cuestión de los males de salud laboral ligados a la autoexplotación, el autopanóptico y al management de la vida a ritmo emprendedor. Esto sucede tanto en los espacios de trabajo, donde las encuestas de salud laboral detectan un incremento de la intensificación, la carga mental, el trabajo emocional, el aislamiento, etc., como en el espacio vital, donde se instala una precariedad generalizada que golpea a la subjetividad en forma de fatiga emprendedora (Han, 2012; Chicchi, 2012).

En segundo lugar, los efectos del tipo emprendedor sobre los modelos de carrera, no lineales, nómadas, particularmente arduos en los grupos de jóvenes universitarios, que soportan los índices más intensos de carreras desterritorializadas, discontinuas y de competición generalizada (Muñoz y Santos, 2017). Con esta última, el nuevo capitalismo parece haber descubierto el secreto para encontrar a la persona de talento entre mil aspirantes, pero lo que no parece saber es qué pasará con los que quedan fuera. Una gran masa de jóvenes titulados esperan aparcados en la tecnópolis emprendedora, donde se forma un ejército de reserva informacional multiusos, dispuesto a la explotación a través del trabajo gratuito, el hope labour, como vía para desarrollar su vocación en un espacio que se interpreta por parte de la empresa como fermento del emprendedor.

\section{Referencias bibliográficas}

Abdelnour, S. (2017). Moi, petite entreprise. Les auto-entrepreneurs, de l'utopie à la réalité, Paris: PUF.

Alonso, L. E. y Fernández Rodríguez, C. (2013). «Los discursos del management. Una perspectiva crítica», Lan Harremanak: Revista de Relaciones Laborales. $\mathrm{n}^{\circ}$ 28: 42-69.

Alter, N. (2011). «Comment les dirigeants des organisations peuvent tuer l'innovation?». Gestion n ${ }^{\circ}$ 36: 5-10.

Altucher, J. (2013). Choose yourself, CreateSpace Independent Publishing Platform.

Boltanski, L. y Chiapello, E (2002). El nuevo espíritu del capitalismo. Madrid: Akal.

Bosma, N., Stam, E. y Wennekers, S. (2012). «Entrepreneurial Employee Activity: A Large Scale International Study», Working Papers n 12-12. Utrecht School of Economics. 
Bouchard, V. y Fayolle, A. (2011). «Comment mettre en oeuvre l'intrapreneuriat?», Gestion. $n^{\circ} 36: 11-21$.

Briales, A. (2017). «Emprendeudores fracasados: individualización neoliberal en los discursos sobre el desempleo». Recerca, Revista de Pensament i Anàlisi. no 20: 79-104.

Brieva, M. (2015). Lo que me está pasando: diarios de un joven emperdedor. Barcelona: Reservoir Books.

Bröckling, U. (2015). El Self Emprendedor. Sociología de una forma de subjetivación. Santiago de Chile: Ediciones Universidad Alberto Hurtado.

Cadar, O. y Badulescu, D. (2015). «Entrepreneur, Entrepreneurship and Intrapreneurship. A Literature Review». (En línea) https://mpra.ub.uni-muenchen.de/78871/1/MPRA_paper_78871.pdf [consulta: 17 de noviembre de 2017]

Chicchi, F. (2012). «Lavoro in frantumi: sofferenza e desiderio nell'epoca della precarietà generalizzata, en Giorgetti, M. y Chicchi, F (ed). Il tempo de la precarietà. Sofferenza soggetiva e disagio della postmodernità (pp.117-136). Milano: Mimesis.

Centro Internacional Santander Emprendimiento (CISE) (2017). Emprendimiento corporativo en España. Gacelas y elefantes bailan sin pisarse (en línea) http://cise.es/gem/ resumen-ejecutivo-informe-emprendimiento-corporativo.pdf [consulta: 16 de noviembre 2017]

Crabtree, S. (2013). "Worldwide, 13\% of Employees Are Engaged at Work" (en línea) http:// news.gallup.com/poll/165269/worldwide-employees-engaged-work.aspx [consulta: 11 de noviembre de 2017]

Davies, W. (2016). La industria de la felicidad. Barcelona: Malpaso

Deleuze, G. (1999). Conversaciones 1972-1990. Valencia: Pretextos.

Douglas, E. y Fitzsimmons, J. (2013). «Intrapreneurial intentions versus entrepreneurial intentions: distinct constructs with different antecedents». Small Business Economics. 41(1): 115-132.

Duvoux, N. (2015). Les oubliés du rêve américain. Philanthropie, État et pauvreté urbaine aux Etats-Unis. Paris: Presses universitaires de France.

Feher, M. (2007). "S'apprécier, ou les aspirations du capital humain", Raisons politiques. $\mathrm{n}^{\circ}$ 28 (4): 11-31.

Feher, M. (2014). "La condición neoliberal. Crédito, autoestima y vínculo". La Maleta de PortBou. n ${ }^{\circ} 6$ julio-agosto 2014.

Feher, M. (2017). Le temps des investis. Essai sur la nouvelle question sociale. Paris: Éditions La Découverte.

Filion, L. J. (2016). «Êtes-vous un intrapreneur?». Gestion. n 41: 100-103.

Foucault, M. (2007). Nacimiento de la biopolítica: Curso del Collège de France (19781979). Buenos Aires: Fondo de Cultura Económica.

Fumagalli, A. (2010). Bioeconomía y capitalismo cognitivo. Hacia un nuevo paradigma de acumulación. Madrid: Traficantes de sueños.

Gorz, A. (2001). «La personne devient une entreprise»: Note sur le travail de production de soi». Revue du MAUSS, n 18 (2), 61-66.

Gorz, A. (2003). L'Immatériel. Connaissance, valeur et capital. Paris: Éditions Galilée.

Han, B. C. (2012). La sociedad del cansancio. Barcelona: Herder.

Hernandez, E. M.(2006). «Extension du domaine de l'entrepreneur... et limites». La Revue des Sciences de Gestion. n²19: 17-26.

Laval, C. y Dardot, P. (2013). La nueva razón del mundo: Ensayo sobre la sociedad neoliberal. Barcelona: Gedisa.

Linhart, D. (2015). La comédie humaine au travail. Toulouse: Éditions Érès. 
Lisein, O. y Degré, J. (2011). «Comprendre les pratiques d'entreprises en matière d'intrapreneuriat». Revue internationale P.M.E. $n^{\circ} 242$, p.167-210.

Lorusso, S. (2016). "Precarity Feels". The Entreprecariat. (en línea) http://networkcultures. org/entreprecariat/precarity-feels/ (consulta 3 de noviembre de 2017)

Mack, M. (2001). «Peut-on apprendre à être intrapreneur? Les leçons d'une expérience», Gestion. $\mathrm{n}^{\circ}$ 26: 10-11.

Marazzi, C. (2003). El sitio de los calcetines. El giro lingüistico de la economía y sus efectos sobre la politica. Madrid: Akal.

McGoey, L. (2014). «The Philanthropic State: market-state hybrids in the philanthro-capitalist turn». Third World Quarterly. 35(1): 109-125.

Menger, P.-M. (2003). Portrait de l'artiste en travailleur. Métamorphoses du capitalisme, Paris: La République des idées, Seuil.

Moruno, J. (2015). La fábrica del emprendedor. Madrid: Ediciones Akal.

Muñoz Rodríguez, D. y Santos Ortega, A. (2017). "Las cárceles del capital humano: trabajo y vidas precarias en la juventud universitaria". Recerca, Revista de Pensament i Anàlisi. $\mathrm{n}^{\circ} 20: 59-78$.

Nicoli, M. (2015). Le risorse umane. Roma: Ediesse.

Nicoli, M. (2017). "Intellettuali di sé. Ambivalenza della start-up esistenziale". Operaviva. 25 abril 2017 (en línea) http://operaviva.info/intellettuali-di-se/ (consulta: 15 de noviembre de 2017).

Pinchot G. III (1985). Intrapreneuring, New York: Harper and Row.

Pongratz, H. J.; Voß, G. G. (2003). «From employee to 'entreployee'. Towards a 'self-entrepreneurial' work force?» Concepts \& Transformation. Vol. 8 (3): 239-254.

Santos Ortega, A. (2014). «La política en manos de los empresarios: el imparable ascenso de la ideología del emprendedor», Papeles de Relaciones Ecosociales y Cambio Global. $\mathrm{n}^{\circ}$ 127: 21-43.

Serrano Pascual, A. (2016) Colonización política de los imaginarios del trabajo: la invención paradójica del 'emprendedor'. En E. Gil Clavo (ed.) (2016) Sociólogos contra el economicismo (pp.112-130) Madrid: Libros de la Catarata

Vega, J. M. (2016). Intraemprendedores. Reinventa tu empresa con espíritu start-up. Barcelona: Libros de cabecera.

Vercellone, C. (2009). "Lavoro, distribuzione del reddito e valore nel capitalismo cognitivo. Una prospettiva storica e teorica". Sociologia del Lavoro. n 115: 31-53. 